\title{
Modified SVPWM Algorithm for Three Level VSI With Synchronized and Symmetrical Waveforms
}

\author{
Abdul Rahiman Beig, Member, IEEE, G. Narayanan, Member, IEEE, and V. T. Ranganathan, Senior Member, IEEE
}

\begin{abstract}
The objective of the present work is to improve the output waveform of three level inverters used in high-power applications, where the switching frequency is very low. This is achieved by maintaining the synchronization, half-wave symmetry, quarter-wave symmetry, and three-phase symmetry in the pulsewidth modulation (PWM) waveforms. The principles of achieving synchronization and symmetries in terms of space vectors for three level inverters are presented. A novel synchronized space vector pulsewidth modulation (SVPWM) algorithms is proposed and verified experimentally. The experimental waveforms of the inverter output voltage and motor no load current for different operating conditions of the drive are presented. The performance measure in terms of the weighted total harmonic distortion $(T H D)$ of the line voltage is computed for the linear modulation region of the drive for the proposed algorithm and compared with that of synchronized SVPWM and synchronized sine-triangle pulsewidth modulation (SPWM) technique. The comparative results show that consideration of synchronization and symmetry results in improved $T H D$. Another significant feature of the proposed algorithm is that the symmetry and synchronization leads to self-balancing of the direct current ( $\mathrm{dc}$ ) bus capacitor voltages over every one third cycle of the fundamental.
\end{abstract}

Index Terms-Harmonic distortion, induction motor drives, pulsewidth modulated inverters, pulsewidth modulation.

\section{INTRODUCTION}

$\mathbf{T}$ HREE level inverters have certain advantages over conventional two level inverters [1].

- Three level inverters can synthesize double the voltage levels using the devices of similar voltage rating. Hence, the power handling capacity can be doubled.

- For a given switching frequency, three level voltage can have double the bandwidth.

- Three level inverters have improved total harmonic distortion $(T H D)$ compared to two level inverters.

Because of these features, three level inverters are finding application especially in medium voltage high-power drives.

In order to reduce the switching losses, the switching frequency $\left(f_{s w}\right)$ of the high-power inverters is limited to low values $(350 \mathrm{~Hz}$ to $1 \mathrm{KHz})$. Hence, the pulse number $(P)$, defined as

Manuscript received December 6, 2004; revised June 20, 2006. Abstract published on the Internet November 30, 2006. This work was published in part in the following: A. R. Beig, G. Narayanan, and V. T. Ranganathan, "Space vector based synchronized PWM algorithm for three level voltage source inverters: Principles and applications to $v / f$ drives," Proc. 28th IEEE Int. Conf. Ind. Electron., IECON'02, pp. 1249-1254, Seville, Spain, November 9-14, 2002.

A. R. Beig is with the Department of Electrical and Electronics Engg. National Institute of Technology Suratkal Srinivasnagar, 575025, Karnataka, India (e-mail: arbeig@ieee.org).

G. Narayanan and V. T. Ranganathan are with the Department of Electrical Engineering, Indian Institute of Science, Bangalore 560012, Karnataka, India. (e-mail: gnar@ee.iisc.ernet.in; vtran@ee.iisc.ernet).

Digital Object Identifier 10.1109/TIE.2006.888801 the ratio of $f_{s w}$ to the fundamental frequency $F_{s}$ is low. Under such circumstances, the output voltage of the inverter will be rich in harmonics [2]-[6]. The output voltage must be synchronized with its fundamental component in order to eliminate subharmonics. Like two level sine-triangle pulsewidth modulation (SPWM), in three level SPWM, with $P$ equal to the odd integer multiple of three, the inverter output waveforms will be synchronized with half-wave symmetry (HWS), quarter-wave symmetry (QWS), three phase symmetry (TPS) [3], [4]. The three level space vector pulsewidth modulation (SVPWM) algorithms for high-power applications use this feature to achieve synchronization and waveform symmetry [5]. In this conventional three level SVPWM scheme, $P$ can take only values $3,9,15 \ldots$ The major drawback of this scheme is that $f_{s w}$ varies over a wide range with $F_{s}$ in variable speed drives.

In the case of conventional two level inverters, it is shown that the flexibility in selecting the space vectors results in design of SVPWM sequences which generate synchronized output waveforms with HWS, QWS, and TPS for any odd integer values of $P$ [6]. The objective of this paper is to exploit similar features of three level space vectors and design SVPWM sequences for three level inverter resulting in synchronized PWM outputs waveforms with HWS, QWS, and TPS. No such attempt is made in the literature in this direction and for the first time a novel synchronized three level SVPWM algorithm with waveform symmetries is proposed for low-switching frequency applications. Even though three level SVPWM is an active area of research, most of the work is focussed on specific issues related to conventional symmetrical SVPWM algorithm like simplifying the algorithm [7], implementation issues [8]-[11], reducing the switching losses [12], neutral point voltage balancing [13]-[18], or reducing common mode voltage [19].

The computational complexity of three level SVPWM can be reduced to that of two level SVPWM, as shown in [7] and is applied to conventional SVPWM algorithm. In the present work, the simplified method given in [7] is further modified so that computation of synchronized SVPWM sequences and implementation on digital controller using assembly program is simple and modular. In Section II, this modified simple approach to SVPWM is explained.

The design of the three level SVPWM sequences require different approach compared to two level SVPWM techniques as there are additional redundancies in space vectors, zero vector is no longer the common vector for all the regions of space vector space and direct current (dc) bus balancing has to be maintained. The basic principle of the space vector approach to synchronization and symmetry and design of sequences is detailed in Section III. The present work shows that by proper design of SVPWM sequences it is possible to get synchronization and various waveform symmetries for any integer 


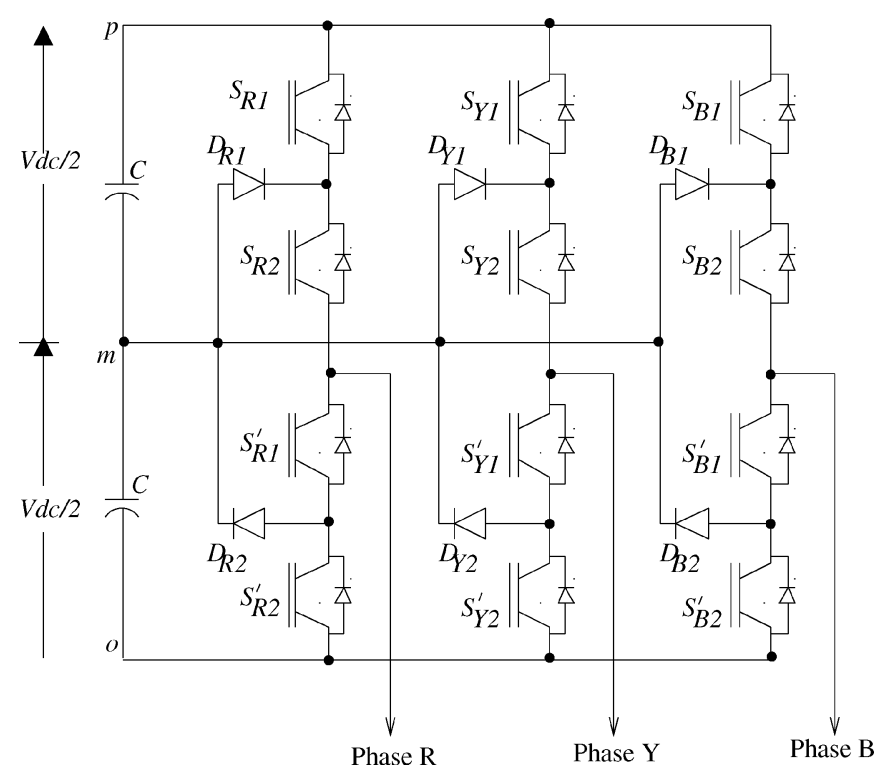

Fig. 1. Three level diode clamp inverter.

values of $P$. The proposed PWM technique is implemented on TMS320F240 fixed point DSP controller. The proposed PWM technique is verified experimentally on three level diode clamp voltage source inverter fed $v / f$ induction motor drive. The implementation details and typical experimental results are presented in Section IV.

The performance of the proposed technique is studied over the entire linear modulation region of the drive. A comparison in terms of the weighted $T H D$ of the output line voltage ( $V_{\text {LWTHD }}$ ) for the proposed synchronized SVPWM technique with waveform symmetries and conventional synchronized SVPWM technique shows that consideration of symmetry results in improved THD. Similarly, a comparison of $V_{\text {LWTHD }}$ of proposed synchronized SVPWM with waveform symmetries and synchronized SPWM technique with waveform symmetries proves the superiority of the present method over synchronized SPWM technique. Also the variation of $V_{\text {LWTHD }}$ as a function of $P$ is studied and this result can be used for the optimal design of PWM technique in order to achieve minimum THD.

A major requirement of three level PWM sequences is that the dc bus capacitor voltages must be balanced. It is shown that the proposed technique ensures the dc link capacitor voltage balancing over (1/3)rd cycle of the fundamental. This is an important result because the proposed method is simple, do not require any additional computation or feed back signal compared to other methods presented in the literature [13]-[18]. The proposed method also results in minimum common mode voltage.

Compared to the other three level SVPWM algortihms, the present work has a unique feature that it addresses all the major issues related to the three level PWM techniques such as computational complexity, synchronization, THD, dc bus voltage balancing, and common mode voltage. Hence, the proposed SVPWM method will be suitable for high-power applications as it eliminates subharmonics by maintaining synchronization, improves $T H D$ through various waveform symmetries, results in balanced dc bus voltage, and low common mode voltage.
TABLE I

Diode Clamp InVERTER: Switch Status AND DEFINITION OF STATE FOR POLE $R$

\begin{tabular}{ccc}
\hline Switch status & State & Pole voltage \\
\hline$S_{R 1}=O N, S_{R 2}=O N$, & & \\
$S_{R 1}^{\prime}=O F F, S_{R 2}^{\prime}=O F F$, & +1 & $V_{R m}=\frac{V_{d c}}{2}$ \\
$D_{R 1}=O F F, D_{R 2}=O F F$ & & \\
\hline$S_{R 1}=O F F, S_{R 2}=O N$, & & \\
$S_{R 1}^{\prime}=O N, S_{R 2}^{\prime}=O F F$, & 0 & $V_{R m}=0 V$
\end{tabular}

$D_{R 1}$ or $D_{R 2}$ will conduct depending on

\begin{tabular}{l} 
the polarity of the load current \\
\hline$S_{R 1}=O F F, S_{R 2}=O F F$, \\
$S_{R 1}^{\prime}=O N, S_{R 2}^{\prime}=O N$, \\
$D_{R 1}=O F F, D_{R 2}=O F F$
\end{tabular}

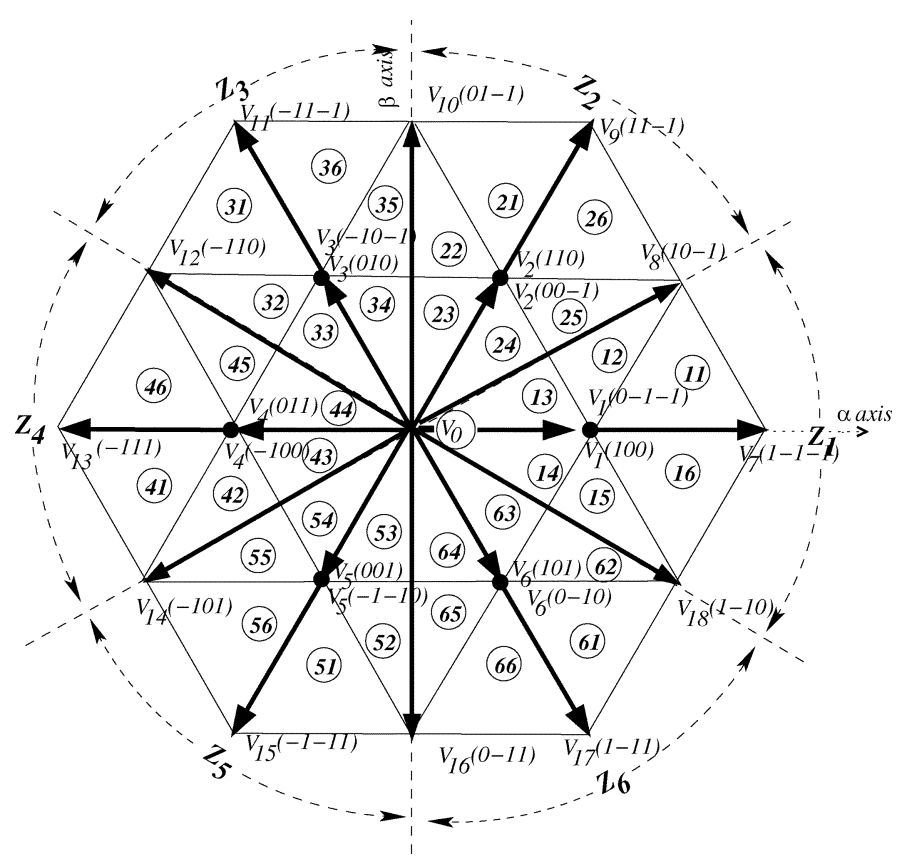

Fig. 2. Space vectors of three level inverter with sector and subsector definition.

\section{SIMPLIFIED APPROACH TO SVPWM}

Fig. 1 shows the circuit diagram of a three level diode clamp inverter. The states are defined in Table I. The space vectors associated with the three level inverters on $\alpha-\beta$ plane are shown in Fig. 2. In the space vector approach to PWM, the reference vector is sampled at regular interval $T_{s}$. The sampled reference vector $\bar{V}_{r}$ is approximated by time averaging the nearest three vectors, $\bar{V}_{x}, \bar{V}_{y}$, and $\bar{V}_{z}$ according to (1) and (2)

$$
\begin{aligned}
\bar{V}_{r} T_{s} & =\bar{V}_{x} T_{x}+\bar{V}_{y} T_{y}+\bar{V}_{z} T_{z} \\
T_{s} & =T_{x}+T_{y}+T_{z}
\end{aligned}
$$

where $T_{x}, T_{y}$, and $T_{z}$ are the dwell times of $\bar{V}_{x}, \bar{V}_{y}$, and $\bar{V}_{z}$, respectively.

Unlike two level inverters, the zero vector is no longer common for all the regions. So solution to (1) and (2) involves solving three simultaneous equations. In order to simplify 


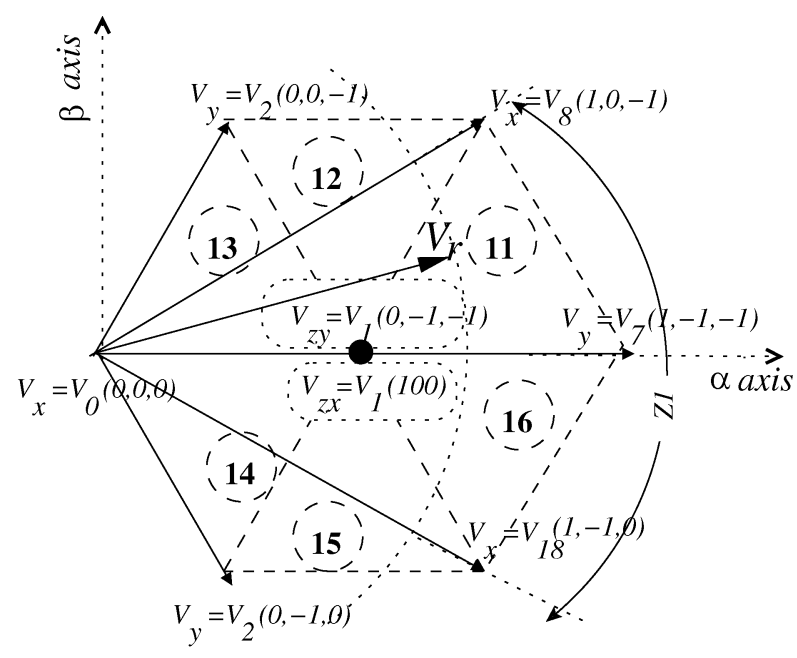

(a)

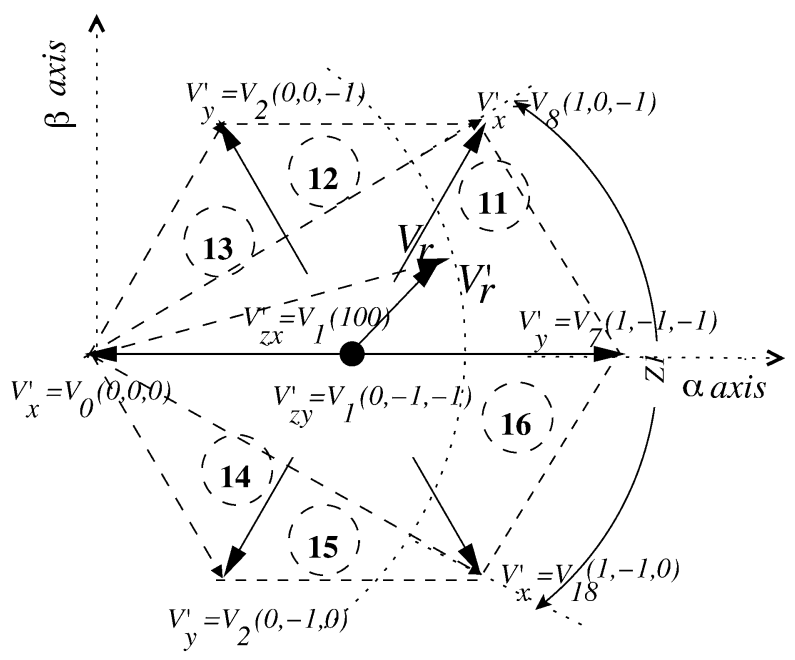

(b)

Fig. 3. (a) Vectors of sector 1. (b) Mapping of vectors of sector 1 to fictitious vectors.

the above equations, a simple approach based on the method given in [7] is adopted, in which the symmetry of the space vectors is exploited. The space vector plane is divided in to six sectors, each of $60^{\circ}$, as shown in Fig. 2. Each sector $Z$, where $Z=1,2,3, \ldots, 6$, is associated with one pivot vector $\bar{V}_{z}$ and six other vectors. The pivot vector $\bar{V}_{1}$ and other six vectors of sector 1 are redrawn in Fig. 3(a). The vectors of the other sectors are phase displaced by $\pi / 3$ radians. All the six sectors exhibit symmetry. All the vectors associated with the given sector $Z$, can be mapped to a set of seven fictitious vectors with $\bar{V}_{1}$ as the center as defined by (3). This is illustrated in Fig. 3(b)

$$
\begin{aligned}
& \bar{V}_{r}^{\prime}=\bar{V}_{r} e^{j(Z-1) \pi / 3}-\bar{V}_{1} \\
& \bar{V}_{x}^{\prime}=\bar{V}_{x} e^{j(Z-1) \pi / 3}-\bar{V}_{1} \\
& \bar{V}_{y}^{\prime}=\bar{V}_{y} e^{j(Z-1) \pi / 3}-\bar{V}_{1} \\
& \bar{V}_{z}^{\prime}=\bar{V}_{z} e^{j(Z-1) \pi / 3}-\bar{V}_{1}
\end{aligned}
$$

The fictitious vectors are similar to those of two level inverters.
TABLE II

Pivot Vectors AND INVERTER STATES

\begin{tabular}{cccc}
\hline Sector & Pivot Vector & $\bar{V}_{z x}$ & $\bar{V}_{z y}$ \\
$Z$ & $\bar{V}_{z}$ & & \\
\hline 1 & $\bar{V}_{1}$ & 100 & $0-1-1$ \\
2 & $\bar{V}_{2}$ & $00-1$ & 110 \\
3 & $\bar{V}_{3}$ & 010 & $-10-1$ \\
4 & $\bar{V}_{4}$ & -100 & 011 \\
5 & $\bar{V}_{5}$ & 001 & $-1-10$ \\
6 & $\bar{V}_{6}$ & $0-10$ & 101 \\
\hline
\end{tabular}

The vector $\bar{V}_{z}^{\prime}$ forms the origin and its magnitude is always zero and for a given sector this vector is similar to the zero vector of two level inverters. The three nearest vectors can be identified as $\bar{V}_{z}^{\prime}, \bar{V}_{x}^{\prime}$, and $\bar{V}_{y}^{\prime}$ as shown in Fig. 3. Now the solution to (1) is similar to that of two level inverters, as in (4)

$$
\begin{aligned}
V_{r \alpha}^{\prime} T_{s} & =V_{x \alpha}^{\prime} T_{x}+V_{y \alpha}^{\prime} T_{y} \\
V_{r \beta}^{\prime} T_{s} & =V_{x \beta}^{\prime} T_{x}+V_{y \beta}^{\prime} T_{y} \\
T_{z} & =T_{s}-T_{x}-T_{y} .
\end{aligned}
$$

Thus, the computational complexity of three level inverters is reduced to that of two level inverters. Implementation of above method is simple as it requires only the computation of $\bar{V}_{r}^{\prime}$ and is explained in Section IV. With the sector definition given above, all the sectors are symmetric and a modular approach can be used in implementation.

Conventional SVPWM sequences will have switching sequences $\bar{V}_{z x} \Longleftrightarrow \bar{V}_{x} \Longleftrightarrow \bar{V}_{y} \Longleftrightarrow \bar{V}_{z y}$. The $T_{z}$ interval is equally derived between pivot vectors $\bar{V}_{z x}$ and $\bar{V}_{z y}$. The pivot vectors $\bar{V}_{z x}$ and $\bar{V}_{z y}$ are defined in Table II. State $V_{z x}$ is defined as the state of $V_{z}$ obtained by switching only one phase of the inverter from state $V_{x}$. Similarly state $V_{z y}$ is defined as the state of $V_{z}$ obtained by switching only one phase of the inverter from state $V_{y}$. These sequences ensure that in each sampling interval, each of the phases is switched at least once. Also these sequences satisfy following two conditions for minimum switching frequency.

- Condition 1: Only one switch is switched during state transition. That is transition from state 1 to state -1 and vice versa is not allowed.

- Condition 2: The final state of present sample will be the initial state of next sample.

In the next section, these SVPWM sequences are modified to achieve synchronized output waveforms with HWS and TPS.

In the present method, the sector decides the angle through which the reference vector $\bar{V}_{r}$ is to be rotated to get the fictitious reference vector $\bar{V}_{r}^{\prime}$ whereas in the simplified method given in [7], the offset values to be subtracted from $\bar{V}_{r}$ to obtain $\bar{V}_{r}^{\prime}$ has to be stored in the form of lookup table or programmed accordingly. This slight modification helps in developing subroutines to generate switching sequence and helps in implementing in DSP assembly program which is clear from the implementation details given in Section IV. 


\section{SPACE Vector APPROACH TO SyNCHRONIZATION AND SYMMETRY}

In this section, the principles of obtaining synchronization and various symmetry in terms of space vectors is derived.

\section{A. Need for Synchronization and Symmetry}

- Synchronization: At low switching frequencies, it is necessary to maintain perfect synchronization of inverter output voltage with respect to its own fundamental to avoid subharmonics. This is possible if and only if the PWM output pole voltage waveform satisfies the condition given below

$$
\begin{aligned}
& v_{R m}(\theta \pm 2 \pi)=v_{R m}(\theta) \\
& v_{Y m}(\theta \pm 2 \pi)=v_{Y m}(\theta) \\
& v_{B m}(\theta \pm 2 \pi)=v_{B m}(\theta)
\end{aligned}
$$

where $\theta$ is any arbitrary angle measured from the reference axis. This can be achieved if the same inverter state is switched at $\theta$ and $(\theta \pm 2 \pi)$ and the dwell time of these states must be equal. This demands the $\bar{V}_{r}$ to be sampled at $\theta$ and $(\theta \pm 2 \pi)$, which is possible only if there are integral number of samples per cycle of the fundamental.

- TPS: At low switching frequency, lower order harmonics are dominant. The PWM sequences should be designed to eliminate some of these harmonics. The three-phase symmetry will ensure that all the harmonics and the fundamental of all the three phases will be perfectly balanced. So the triplen harmonics will be cancelled from the line voltage. For a phase sequence of $R-Y-B$, the TPS can be achieved if the inverter output voltages of three phases satisfy the following condition:

$$
v_{B m}\left(\theta-\frac{2 \pi}{3}\right)=v_{Y m}\left(\theta+\frac{2 \pi}{3}\right)=v_{R m}(\theta) .
$$

The above condition can be met if the switch position of pole $R$ at $\theta$, the switch position of pole $Y$ at $(\theta+2 \pi / 3)$, and the switch position of pole $B$ at $(\theta-2 \pi / 3)$ are same. For example, if the inverter state at $\theta$ is $(101)$, then at $(\theta+2 \pi / 3)$ the state (110) must be used and at $(\theta-2 \pi / 3)$ state $(011)$ must be used to achieve TPS and dwell time of these states must be equal.

- HWS: The half wave symmetry will ensure elimination of even harmonics from the output voltage. In order to achieve HWS, the pole voltage at $\theta$ and $(\pi+\theta)$ should have opposite polarity that is

$$
\begin{aligned}
& v_{R m}(\theta \pm \pi)=-v_{R m}(\theta) \\
& v_{Y m}(\theta \pm \pi)=-v_{Y m}(\theta) \\
& v_{B m}(\theta \pm \pi)=-v_{B m}(\theta) .
\end{aligned}
$$

In order to achieve this, the switch position of a given phase at $\theta$ and $(\theta \pm \pi)$ must be opposite. If at $\theta$ the switch position of a given phase is " 1 ," then at $(\theta \pm \pi)$ it must be " -1 " and vice versa. But if the switch position of a given phase is " 0 " at $\theta$, then the switch position of the same phase must be " 0 " at $(\theta \pm \pi)$.

The conditions of waveform symmetry for a given sample $\mathrm{k}$ are summarized in Table III. The necessary and sufficient conditions in terms of the inverter states to achieve synchronization
TABLE III

CONDITIONS OF SYNCHRONIZATION, HWS, AND TPS IN TERMS OF POLE VOLTAGES

\begin{tabular}{ccccc}
\hline & Synchronization & HWS & TPS & TPS \\
\hline Pole voltage & Pole voltage & Pole voltage & Pole voltage & Pole voltage \\
at $\theta$ & at $\theta \pm 2 \pi$ & at $\theta \pm \pi$ & at $\theta+2 \pi / 3$ & at $\theta-2 \pi / 3$ \\
\hline$v_{R m}$ & $v_{R m}$ & $-v_{R m}$ & $v_{B m}$ & $v_{Y m}$ \\
$v_{Y m}$ & $v_{Y m}$ & $-v_{Y m}$ & $v_{R m}$ & $v_{B m}$ \\
$v_{B m}$ & $v_{B m}$ & $-v_{B m}$ & $v_{Y m}$ & $v_{R m}$ \\
\hline
\end{tabular}

TABLE IV

CONDITIONS OF SYNCHRONIZATION, HWS, AND TPS IN TERMS OF INVERTER STATES

\begin{tabular}{ccccc}
\hline & Synchronization & HWS & TPS & TPS \\
\hline $\begin{array}{cccc}\text { Inverter state } \\
\text { at } \theta\end{array}$ & Inverter state & Inverter state & Inverter state & Inverter state \\
\hline$S_{R}$ & at $\theta \pm 2 \pi$ & at $\theta \pm \pi$ & at $\theta+2 \pi / 3$ & at $\theta-2 \pi / 3$ \\
$S_{Y}$ & $S_{R}$ & $S_{R}^{\prime}$ & $S_{B}$ & $S_{Y}$ \\
$S_{B}$ & $S_{Y}$ & $S_{Y}^{\prime}$ & $S_{R}$ & $S_{B}$ \\
\hline
\end{tabular}

and symmetry at the line and pole voltages are given in Table IV. In Tables III and IV, the states with a prime $\left(^{\prime}\right)$ indicate the complementary states. Complementary state of 1 is state -1 and vice versa and complementary state of 0 is state 0 itself. The conditions of HWS and TPS given in column 2 and 3 of Table IV, relate the inverter states over an interval of $60^{\circ}$. So the inverter states of each sector are related by (5)

$$
\begin{aligned}
& S_{R}\left(\theta+\frac{\pi}{3}\right)=S_{Y}^{\prime}(\theta) \\
& S_{Y}\left(\theta+\frac{\pi}{3}\right)=S_{B}^{\prime}(\theta) \\
& S_{B}\left(\theta+\frac{\pi}{3}\right)=S_{R}^{\prime}(\theta) .
\end{aligned}
$$

So one of the requirements of synchronization and symmetry is that, there should be integral number of samples $(N)$ per sector and these samples should be placed at identical positions in each sector. Under these conditions, the sampled reference voltages satisfy the conditions given in Table IV. Hence, for a given sample $n$, where $n=1,2,3 \ldots N$, the dwell times $T_{x}, T_{y}$ and $T_{z}$ of nearest three vectors will be equal in all the sectors.

\section{B. Number of Samples per Sector, Pulse Number and Switching Sequence}

1) Odd Values of $N$ : For odd values of $N$, there will be $(N-1)$ samples within the sector placed at an equal distance of $(\pi / 3 N)$ and one sample on the sector boundary. Depending on how the change of $\bar{V}_{z}$ is accomplished, there are two possibilities.

- TYPE 1: All the samples except the sample on sector boundary will have sequences $\bar{V}_{z x} \Longleftrightarrow \bar{V}_{x} \Longleftrightarrow$ $\bar{V}_{y} \Longleftrightarrow \bar{V}_{z y}$.

The sample $n=N$ which falls on the sector boundary will have the sequence,

$\bar{V}_{z x} \longrightarrow \bar{V}_{x} \longrightarrow \bar{V}_{y}$. The last state $\bar{V}_{y}$, of the $N$ th sample of the present sector will be the starting state $\bar{V}_{z x}$, in the first sample of the next sector. So the sample $n=1$ 
will have the sequence, $\bar{V}_{z x} \longrightarrow \bar{V}_{x} \longrightarrow \bar{V}_{y} \longrightarrow \bar{V}_{z y}$. There will not be any switching from one sample to another sample during sector change over. The pulse number is given by $P=(3 / 2)(N-1)$.

- TYPE 2: All the samples will have sequences $\bar{V}_{z x} \Longleftrightarrow$ $\bar{V}_{x} \Longleftrightarrow \bar{V}_{y} \Longleftrightarrow \bar{V}_{z y}$.

The sequence of the last sample in a sector will always end with state $\bar{V}_{z y}$ and the first sample in a sector will start with state $\bar{V}_{z x}$. This type of sequences will cause an additional switching during sector change over. The pulse number is given by $P=(3 / 2)(N+1)$.

2) Even Values of $N$ : For even values of $N$, all the samples will be within the sector placed at an equal distance of $(\pi / 3 N)$. All the samples except $n=1$ and $n=N$ will have sequences $\bar{V}_{z x} \Longleftrightarrow \bar{V}_{x} \Longleftrightarrow \bar{V}_{y} \Longleftrightarrow \bar{V}_{z y}$.

The sample $n=1$ and $n=N$ will have the sequences $\bar{V}_{z x} \longrightarrow \bar{V}_{x} \longrightarrow \bar{V}_{y} \longrightarrow \bar{V}_{x}$ and $\bar{V}_{x} \longrightarrow \bar{V}_{y} \longrightarrow \bar{V}_{x} \longrightarrow$ $\bar{V}_{z x}$.

In these samples, one of the phases will be switched twice and another phase will be clamped. The pulse number will be $P=3 N / 2$. Combining all the three cases, it can be seen that synchronization, HWS and TPS can be achieved for any integral value of $P$.

It can be seen that these sequences also exhibit the conditions of QWS given in (6) in addition to the conditions of synchronization, HWS and TPS, even though the sequences are designed without considering conditions of QWS

$$
\begin{aligned}
& S_{R}\left(\theta_{R}+\theta\right)=S_{R}\left(\theta_{R}-\theta\right) \\
& S_{Y}\left(\theta_{Y}+\theta\right)=S_{Y}\left(\theta_{Y}-\theta\right) \\
& S_{B}\left(\theta_{B}+\theta\right)=S_{B}\left(\theta_{B}-\theta\right)
\end{aligned}
$$

where $\theta_{R}, \theta_{Y}$, and $\theta_{B}$ are the instants at which the $\mathrm{R}, \mathrm{Y}$ and $B$ phase fundamental waveforms have their positive or negative peaks. So the output voltages will also have QWS.

\section{EXPERIMENTAL IMPLEMENTATION AND RESULTS}

The conventional space vector modulation algorithm explained in Section II can be modified to incorporate the conditions of synchronization and symmetry. So the modified algorithm is as follows:

1) Identification of sector $Z$ : From the normalized values of $V_{r \alpha}$ and $V_{r \beta}$, the sector $Z$ is identified as follows.

If $V_{r \alpha} \geq 0$ and $V_{r \beta} \geq 0$, then, if $V_{r \beta}>V_{r \alpha} \tan (\pi / 6)$, then $Z=2$, else, $Z=1$.

If $V_{r \alpha} \geq 0$ and $V_{r \beta}<0$, then, if $V_{r \beta}>V_{r \alpha} \tan (\pi / 6)$, then $Z=6$, else, $Z=1$.

If $V_{r \alpha}<0$ and $V_{r \beta} \geq 0$, then, if $V_{r \beta}>\left|V_{r \alpha}\right| \tan (\pi / 6)$, then $Z=3$, else, $Z=4$.

If $V_{r \alpha}<0$ and $V_{r \beta}<0$, then, if $\left|V_{r \beta}\right|>\left|V_{r \alpha}\right| \tan (\pi / 6)$, then $Z=5$, else, $Z=4$.

2) Identification of subsectors: Map $V_{r}$ to fictitious vector $V_{r}^{\prime}$ using the expression $\bar{V}_{r}^{\prime}=\bar{V}_{r} e^{j(Z-1) \pi / 3}-\bar{V}_{1}$. From the $\alpha$ and $\beta$ components of $V_{r}^{\prime}$, determine the sub-sector. Determination of subsector is similar to identifying sectors as

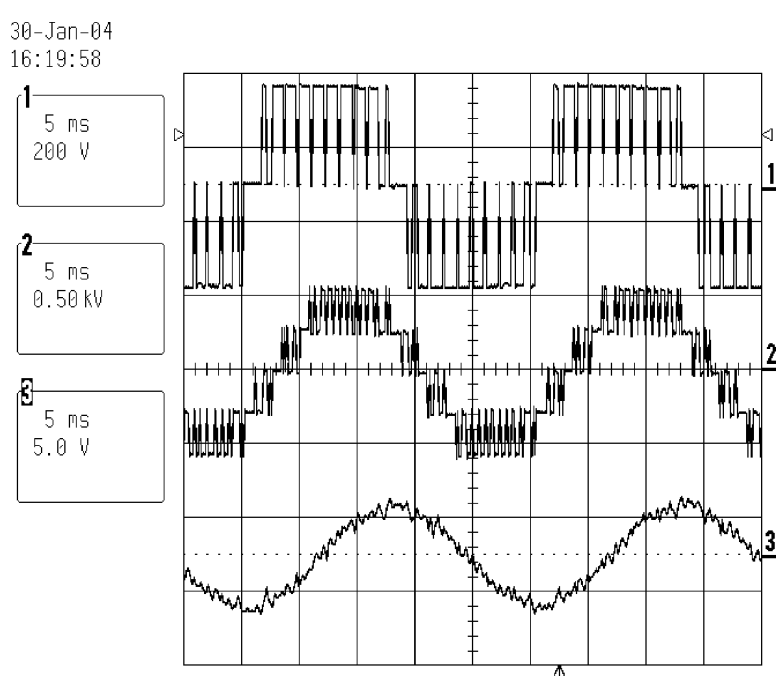

Fig. 4. Experimental waveforms: ch1: $v_{R m}$ (Scale:200 V/div) $\operatorname{ch} 2: v_{R Y}$ (Scale:500 V/div) ch3: $i_{R}\left(\right.$ Scale:11.1 A/div) for $F_{s}=40 \mathrm{~Hz}, N=7$, $P=10$.

explained in step 1 above except that $V_{r \alpha}$ and $V_{r \beta}$ are replace by $V_{r \alpha}^{\prime}$ and $V_{r \beta}^{\prime}$, respectively.

3) Computation of switching intervals $T_{z}, T_{x}$, and $T_{y}$ : From sector, subsector, $V_{r \alpha}^{\prime}$, and $V_{r \beta}^{\prime}$, the $T_{z}, T_{x}$ and $T_{y}$ can be computed using (4).

4) Identification of switching sequence: The $F_{s}$ and $f_{s w}$ will decide the value of $N$. A sector and subsector combination and $N$ will uniquely define the switching sequence and this can be programmed in the form of subroutines.

This can be implemented in another way also. The switching sequences of all the six subsectors of sector 1 are stored in the form of a look up table. Using this the switching sequence of other sectors are computed using (5).

The above algorithm is implemented in TMS320F240 based controller. The event manager is programmed in compare mode. Depending on the value of sector and subsector, the corresponding subroutine is executed and the counters are loaded with proper values. Thus, the switching pulses are generated. The exact sampling time interval is maintained using end of period interrupt of event manager module. The event manager module can generate six independent signals. The complementary signals and the dead time between these signals are generated in the external circuit. This SVPWM algorithm is applied to open loop $v / f$ induction motor drive. The experimental drive consists of a $400 \mathrm{~V}, 10 \mathrm{HP}$, three phase, $50 \mathrm{~Hz}$, induction motor powered from a $30 \mathrm{KVA}$, insulated gate bipolar transistor (IGBT) based three phase three level diode clamp inverter. The dc bus voltage is set at $510 \mathrm{~V}$. The typical experiential results for $F_{s}=40 \mathrm{~Hz}$ is shown in Fig. 4. The harmonic spectra of $V_{R m}, V_{R Y}$ and $i_{R}$, computed from the experimental data is given in Fig. 5.

It can be seen from harmonic spectra of experimental waveforms (Fig. 5) that, the output voltage has no sub harmonics and even harmonics. Hence, the modified SVPWM guaranties synchronization and half-wave symmetry. The harmonic spectra shows that the triplen harmonics which are present in phase 

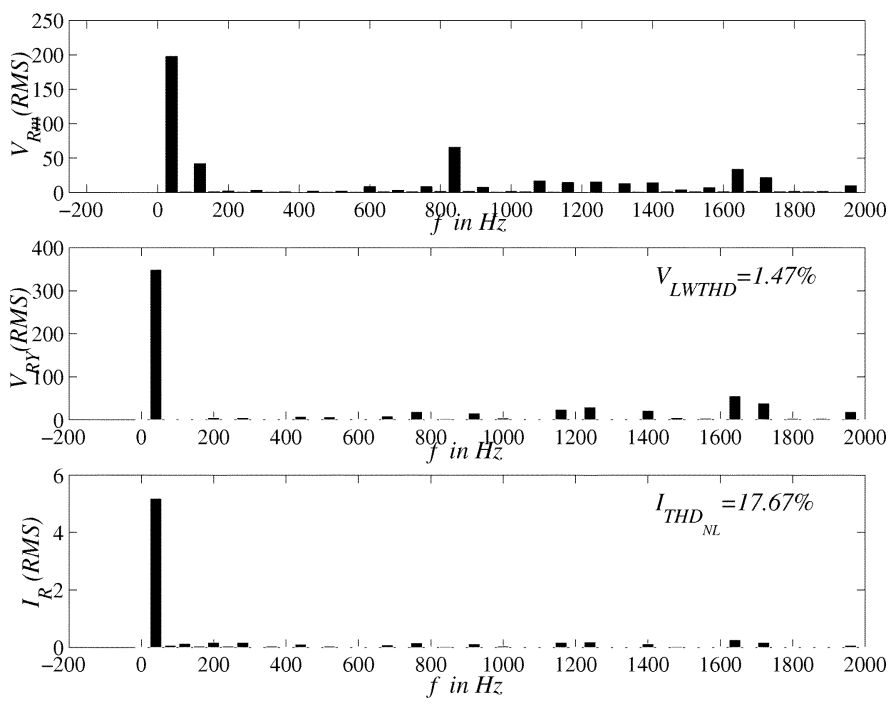

Fig. 5. Harmonic spectrum of $v_{R m}, v_{R Y}$, and $i_{R}$ at $F_{s}=40 \mathrm{~Hz}, N=7$, $P=10$.

voltage are absent from line voltage and motor current. This proves that the SVPWM preserves three phase symmetry.

\section{PERformance ANALYSIS}

The performance of the proposed synchronized SVPWM sequences is studied in terms of the THD of output waveforms, effect of PWM on dc bus midpoint voltage variation and common mode voltage. Some of these results are presented in this section.

\section{A. THD}

In high-power drives, the THD of the motor no load current, $I_{T H D_{N L}}$, which is a function of voltage spectra and motor parameters is a suitable performance index at low switching frequencies [2]. $I_{\mathrm{THD}_{\mathrm{NL}}}$ is defined in (7)

$$
I_{T H D_{N L}}=\frac{\sqrt{\sum I_{n}^{2}}}{I_{1_{N L}}}, n \neq 1
$$

where $I_{1_{N L}}$ is the RMS value of the fundamental component of the motor no load current.

Measuring $I_{T H D_{N L}}$ experimentally is a difficult process. So another quantity which is proportional to $I_{T H D_{N L}}$ and easy to compute, is the weighted $T H D$ of the line voltage ( $\left.V_{\text {LWTHD }}\right)$ [6]. $V_{\text {LWTHD }}$ is defined in (8)

$$
V_{\text {LWTHD }}=\frac{\sqrt{\sum\left(\frac{V_{n}}{n}\right)^{2}}}{V_{1}}, n \neq 1,
$$

The $V_{\text {LWTHD }}$ gives the measure of $I_{\mathrm{THD}_{\mathrm{NL}}}$ as these are related as follows:

$$
V_{\mathrm{LWTHD}}=\frac{L_{l}}{L_{o}} I_{T H D_{N L}}
$$

where $L_{O}$ is the magnetizing inductance and $L_{l}$ is the total leakage inductance. Therefore, $V_{\text {LWTHD }}$ can be used as the measure of $I_{T H D_{N L}}$.
The drive is run at no load at different values of $F_{s}$ in the linear modulation range (i.e., $M_{i}<0.886$, where modulation index, $M_{i}$ is defined as the ratio between the magnitude of reference vector to the dc bus voltage $V_{d c}$ ). In each case, the motor current waveform is stored using high bandwidth digital storage scope (LeCroy make, $200 \mathrm{MHz}, 40000$ points per channel). From this data, exactly one cycle data of the no load current is taken. The frequency components are computed using MATLAB-FFT function. From the harmonic components, the $I_{T H D_{N L}}$ of motor no load current is computed using (7) and its variation is shown in Fig. 6.

Fig. 7 give the plot of $V_{\text {LWTHD }}$ of line voltage with respect to $M_{i}$ for different switching frequencies. This result can be used for the optimal selection of switching frequency to minimize $T H D$. The graph with * mark shows the variation of $V_{\mathrm{LWTHD}}$ for a given switching frequency. In this case, the $f_{s w}$ is varied from 380 to $420 \mathrm{~Hz}$.

Fig. 8 shows the comparison of $V_{\text {LWTHD }}$ of the proposed synchronized SVPWM with HWS and TPS and conventional synchronized SVPWM without HWS and TPS. In both the cases, synchronization is maintained. The proposed synchronized SVPWM with symmetry will result in better THD.

Fig. 9 shows the comparison of $V_{\mathrm{LWTHD}}$ of proposed synchronized SVPWM and synchronized SPWM. In both the cases, $P$ is selected such that synchronization, HWS and TPS is maintained. In the case of SPWM, in order to maintain synchronization, HWS and TPS, $P$ should be odd integral multiple of three. These results show that the proposed synchronized SVPWM sequences result in improved $T H D$. Since the proposed SVPWM guaranties synchronization and symmetry for all integer values of $P$, the overall variation of switching frequency throughout the linear range of modulation is low compared to that of synchronized SPWM.

\section{B. Self Balancing of DC Bus Capacitor Voltage}

In symmetrical SVPWM, in every sample the voltage imbalance during the interval $t_{z x}$ is cancelled by the voltage imbalance in interval $t_{z y}$. In addition to this, because of the synchronization and symmetry considerations, the timing intervals of a given sample $n$ will be equal in all the sectors. Hence, it can be shown that for a given sample $n$, the variation of dc bus midpoint voltage $\Delta V_{m}$ in a sector is equal but opposite to $\Delta V_{m}$ in next sector.

As an example for a balanced three phase load such as three phase induction motor load, with phase sequence R-Y-B, power factor of $\phi$ and dc bus capacitor $C$, the dc bus mid point voltage imbalance at interval 1 of sample 1 in sector 1 , is given by

$$
\begin{aligned}
\Delta V_{m 1} & =\frac{1}{C} \int_{\pi / 3}^{(\pi / 3)+\theta_{11}} i_{a 11} d \theta \\
& =\frac{I}{C}\left[2 \cdot \cos \left(\frac{\theta_{11}}{2}\right) \cdot \cos \left(\frac{\pi}{3} \pm \phi+\frac{\theta_{11}}{2}\right)\right]
\end{aligned}
$$

where $I$ is the peak value of the load current, $\theta_{11}$ is the dwell time of the interval 1 of sample 1 and $i_{a 11}$ is the dc bus midpoint current at interval 1 of sample 1 of sector 1 . 


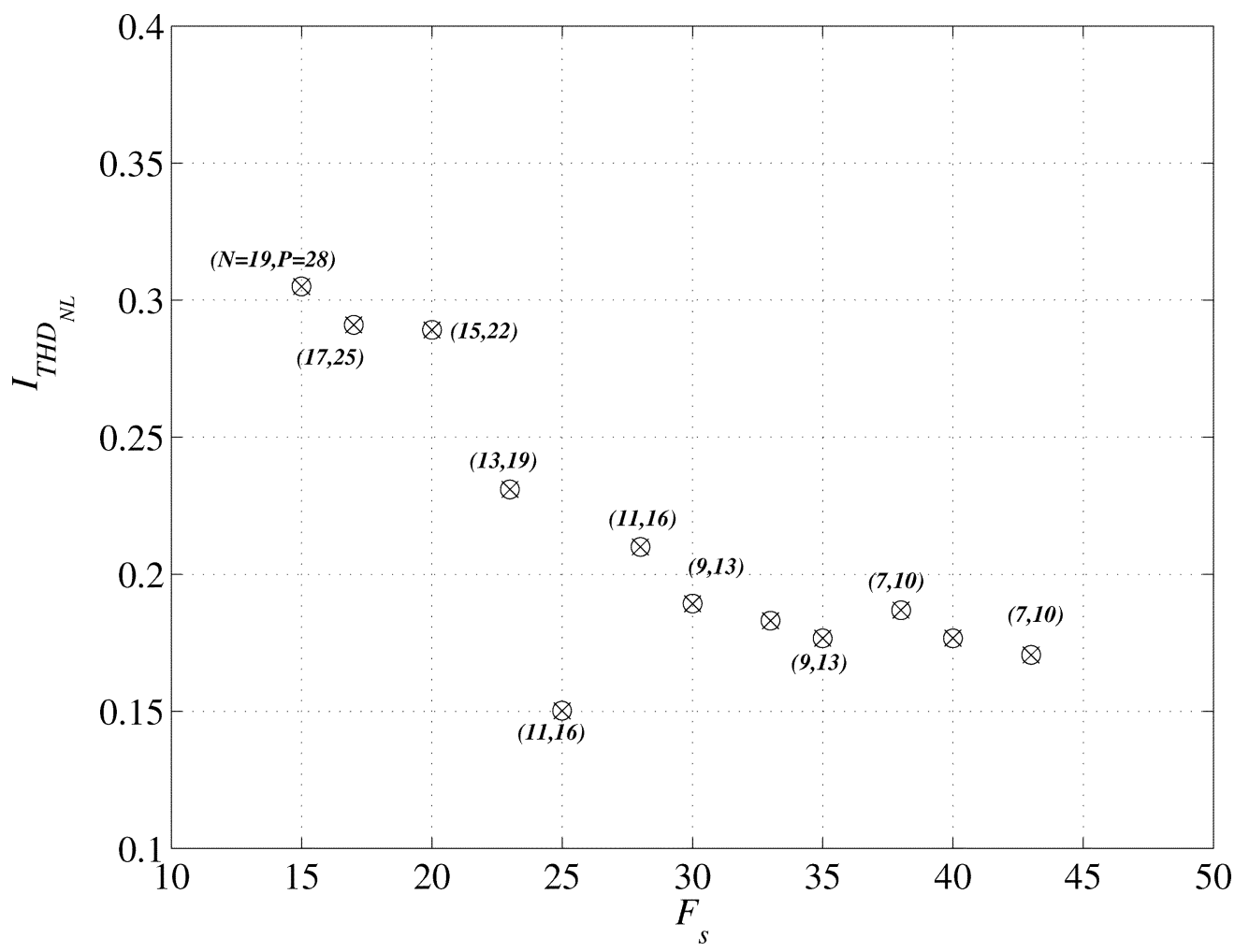

Fig. 6. Experimental results: $I_{T H D_{N L}}$ v/s $F_{s}$.

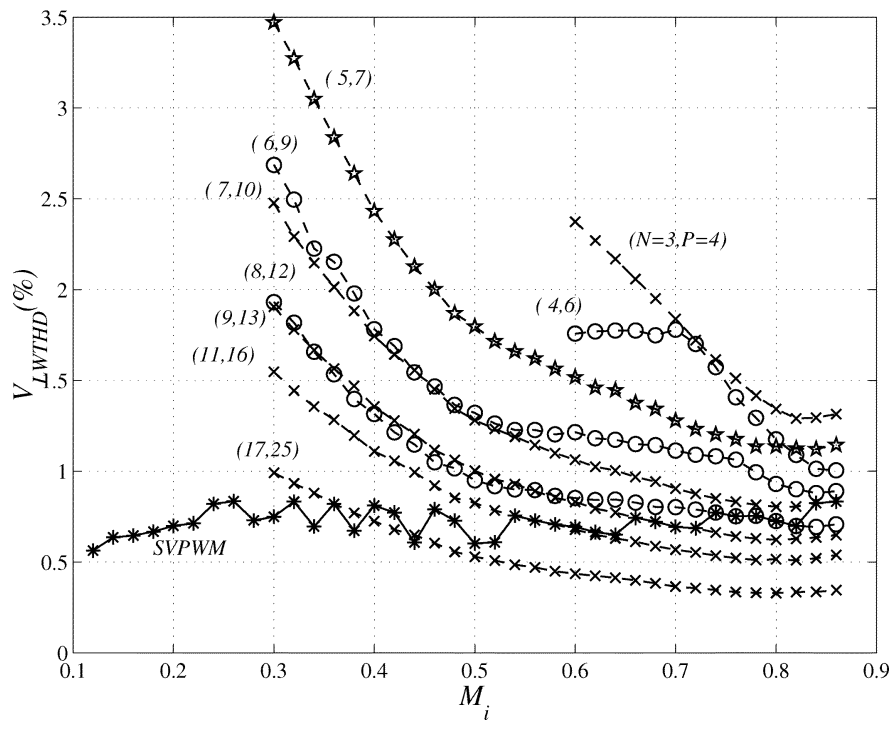

Fig. 7. $V_{\text {LWTHD }}(\%)$ v/s $M_{i}$ plot.

Similarly de mid point voltage imbalance at interval 1 of sample 1 in sector 2 , is given by

$$
\begin{aligned}
\Delta V_{m 2} & =\frac{1}{C} \int_{2 \pi / 3}^{(2 \pi / 3)+\theta_{11}} i_{c_{21}} d \theta \\
& =\frac{I}{C}\left[-2 \cdot \cos \left(\frac{\theta_{11}}{2}\right) \cdot \cos \left(\frac{\pi}{3} \pm \phi+\frac{\theta_{11}}{2}\right)\right]
\end{aligned}
$$

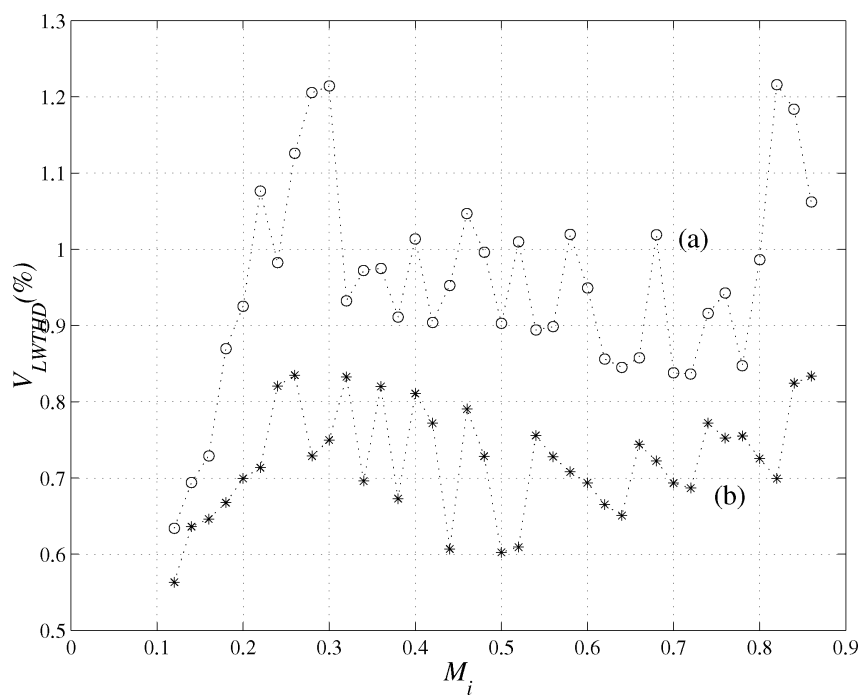

Fig. 8. $\quad V_{\text {LWTHD }}(\%)$ v/s $M_{i}$ plot. (a) SVPWM (synchronized but no symmetry). (b) SVPWM (synchronized with HWS and TPS).

where $i_{c 21}$ is the dc bus midpoint current at interval 1 of sample 1 of sector 2 From above

$$
\Delta V_{m 1}=-\Delta V_{m 2} .
$$

This is true for all values of $n$.

The dc bus capacitor voltage imbalance in one sector will be equal and opposite to that in next sector. Hence, because of symmetry and synchronized approach, the dc bus capacitor voltages are balanced over every $2 \pi / 3$ radians. This is illustrated by the 


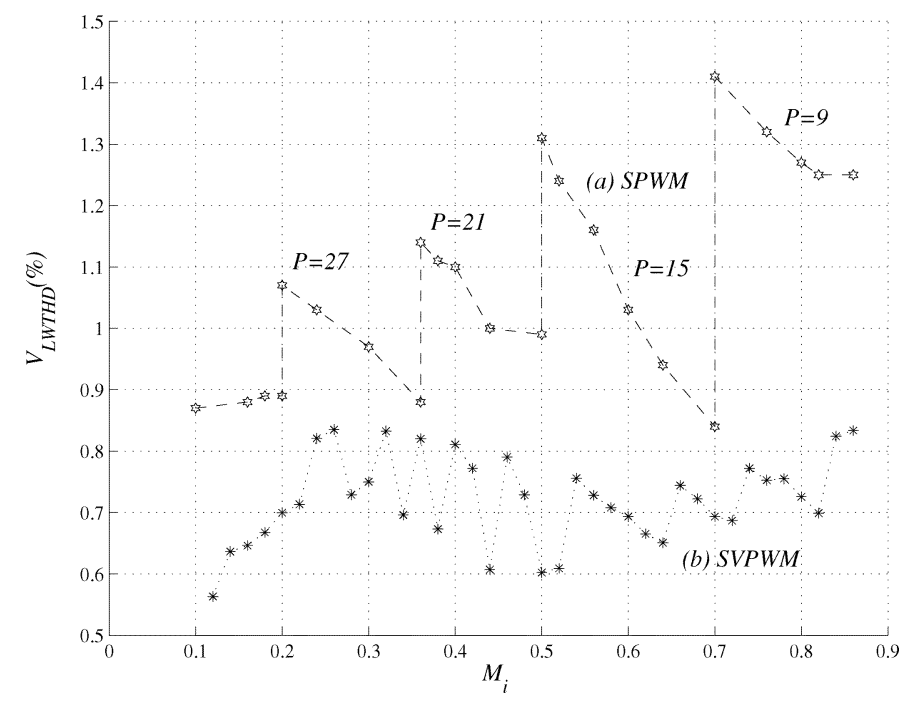

Fig. 9. $V_{\text {LWTHD }}(\%)$ v/s $M_{i}$ plot. (a) SPWM. (b) SVPWM.
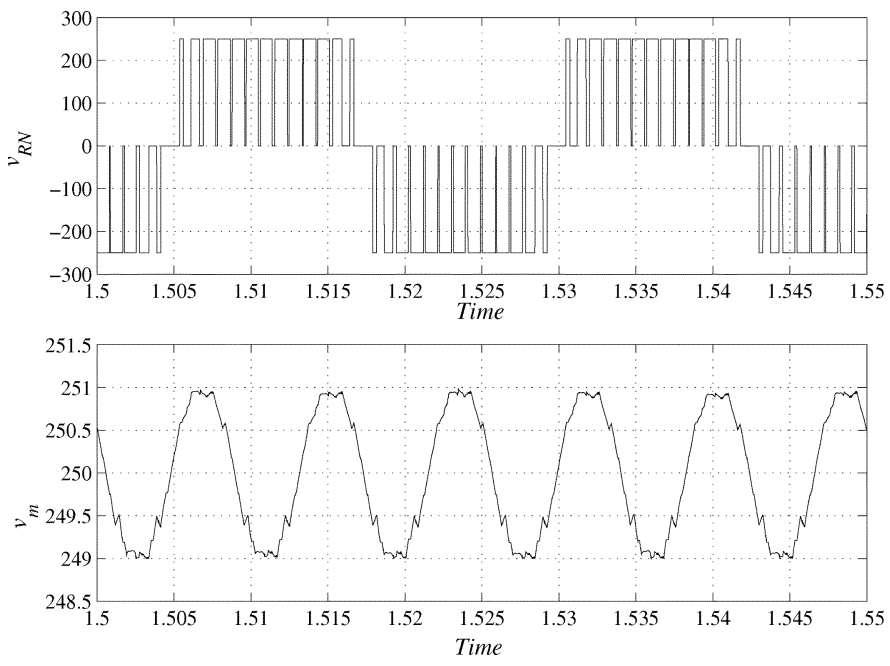

Fig. 10. Simulation results: $v_{R m}$ and dc bus midpoint voltage $v_{m}$ at no load, $F_{s}=40 \mathrm{~Hz}, N=7, P=10, V_{d c}=500 \mathrm{~V}$.

simulation result given in Fig. 10 and verified experimentally, as shown in Fig. 11. This is an important result. Imbalance in dc bus capacitor voltages will result in loss of HWS, thereby even harmonics in motor, even if the sequences are designed to have HWS [13]. Perfect balancing of dc bus capacitor voltages helps in preserving HWS and thus even harmonics in the motor are eliminated. Compare to the other methods [13]-[18], the proposed SVPWM algorithm is simple, does not require any additional feedback signal as in [13] and [14] or0 additional computation as in [16]-[18], and neutral point voltage balance is built in to the algorithm.

\section{Common Mode Voltage}

The proposed SVPWM techniques make use of only V0(000) state; therefore, the maximum value of the voltage between dc bus midpoint $(m)$ and motor neutral point $(n)\left(v_{n m}\right)$, which contributes for the common mode voltage is limited to $(1 / 3) V_{d c}$.

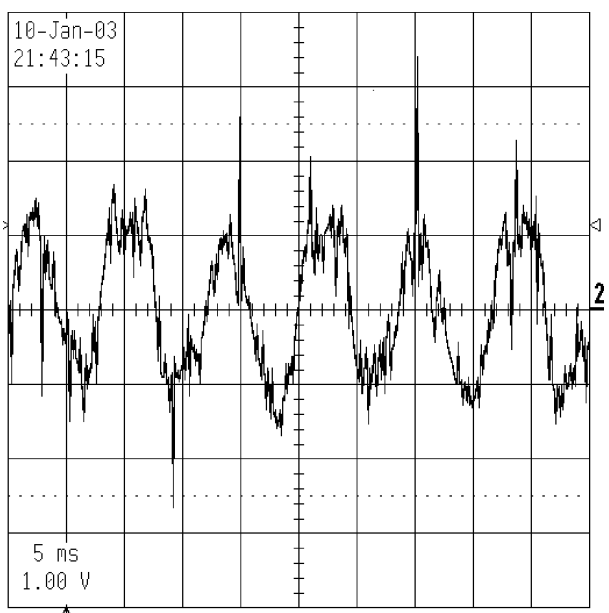

Fig. 11. Experimental waveforms: $\Delta V_{m}$ at no load. (Scale: $\left.1 \mathrm{~V} / \mathrm{div}\right), F_{s}=$ $40 \mathrm{~Hz}, N=7, P=10, V_{d c}=510 \mathrm{~V}$.

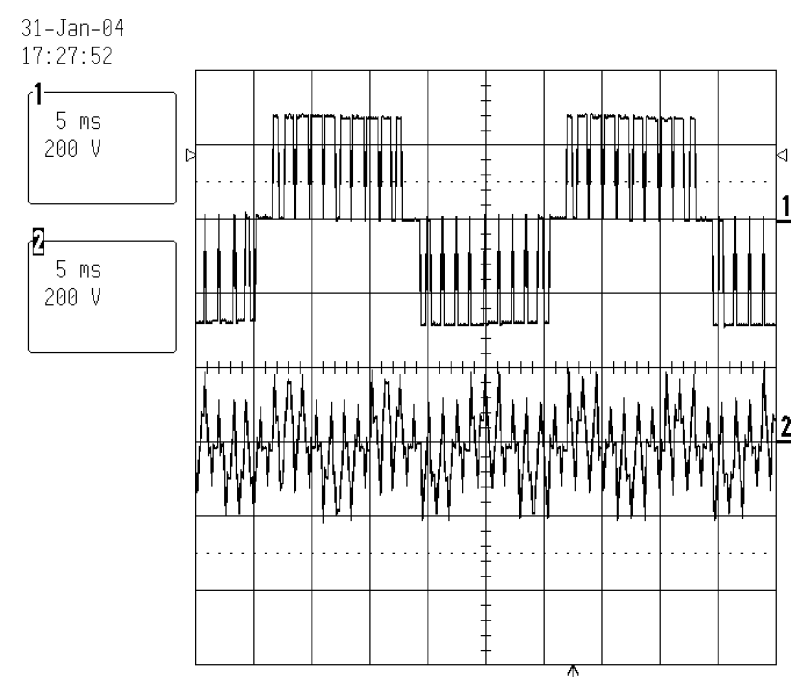

Fig. 12. $v_{n m}$ at $F_{s}=40 \mathrm{~Hz}$. Ch1: $v_{R m}$ (Scale: $200 \mathrm{~V} /$ div), Ch2: $v_{n m}$ (Scale: $200 \mathrm{~V} / \mathrm{div})$.

Also the change in $v_{n m}$ is limited to $(1 / 6) V_{d c}$. The experimental waveforms of the variation of common mode voltage at $F_{s}=40 \mathrm{~Hz}$ is given in Fig. 12.

\section{CONCLUSION}

The theory of the space vector based synchronized PWM method with HWS and TPS is explained. The experimental results are presented. The proposed algorithm is simple to implement on digital controllers and does not add to any computational complexity. It is shown that the proposed PWM technique will result in balanced dc link capacitor voltages. The performance results show that the $V_{\mathrm{WTHD}}$ of the proposed method is better compared to that of synchronized SPWM technique and synchronized SVPWM technique without symmetries. The experimental waveforms show HWS and TPS for any integer value of pulse number. The absence of triplen harmonics from the line voltage shows that the inverter output voltages have threephase symmetry. Even though the focus is on low switching 
frequency applications, the proposed technique can be used for high switching frequency applications also.

\section{REFERENCES}

[1] J.-S. Lai and F. Z. peng, "Multilevel converters-A new breed of power converters," IEEE Trans. Ind. Appl., vol. 32, no. 3, pp. 509-517, May/ Jun. 1996

[2] H. Stemmler, "High-power industrial drives," Proc. IEEE, vol. 82, pp. 1266-1286, Aug. 1994.

[3] J. K. Steinke, "Switching frequency optimal PWM control of a three level inverter," IEEE Trans. Power Electron., vol. 7, no. 3, pp. 487-496, Jul. 1992.

[4] G. Carrara, S. Gardella, M. Marchesoni, R. Salutari, and G. Sciutto, "A new multilevel PWM method: A theoretical analysis," IEEE Trans. Power Electron., vol. 7, no. 5, pp. 497-505, Jul. 1992.

[5] Q. Ge, X. Wang, S. Zhang, Y. Li, and L. Kong, "A high power NPC three level inverter equipped with IGCTs," in Proc. Int. Power Electronand Motion Control Conf. IPEMC, Aug. 14-16, 2004, vol. 3, pp. $1097-1100$.

[6] G. Narayanan and V. T. Ranganathan, "Synchronized PWM strategies based on space vector approach. Part 1: Principles of waveform generation," IEE Proc. Electric Power Appl., vol. 146, no. 3, pp. 267-275, May 1999.

[7] J. H. Seo, C. H. Choi, and D. S. Hyun, "A new simplified space-vector PWM method for three level inverters," IEEE Trans. Power Electron., vol. 16, no. 4, pp. 545-550, Jul. 2001.

[8] S. Chen and G. Joos, "Symemtrical SVPWM pattern generator using field programmable gate array implementation," in Proc. IEEE Appl. Power Electron. Conf., 2002, vol. 2, pp. 1004-1010.

[9] S. Wei, B. Wu, and Q. Wang, "An improved space vector PWM control algorithm for multilevel inverters," in Proc. Int. Power Electron. Motion Control Conf., Aug. 14-16, 2004, vol. 3, pp. 1124-1129.

[10] C. Wang, B. K. Bose, V. Oleschuk, S. Mondal, and J. O. P. Pinto, "Neural-network-based space-vector PWM of a three level inverter covering overmodulation region and performance evaluation on induction motor drive," in Proc. IECON '03 Conf., Nov. 2-6, 2003, vol. 1 , pp. 1-6.

[11] M.-C. Wong, Z.-Y. Zhao, Y.-D. Han, and L.-B. Zhao, "Three-dimensional pulse-width modulation technique in three level power inverters for three-phase four-wired system," IEEE Trans. Power Electron., vol. 16, no. 3, pp. 418-427, May 2001.

[12] T. Bruckner and D. G. Holmes, "Optimal pulse-width modulation for three level inverters," IEEE Trans. Power Electron., vol. 20, no. 1, pp. 82-89, Jan. 2005.

[13] N. Celanovic and D. Borojevic, "A comprehensive study of neutralpoint voltage balancing problem in three level neutral point clamped voltage source PWM inverters," IEEE Trans. Power Electron., vol. 15, no. 2, pp. 242-249, Mar. 2002.

[14] K. Yamanaka, A. M. Hava, H. Kirino, Y. Tanaka, N. Koga, and T. Kume, "A novel neutral point potential stabilization technique using the information of output current polarities and voltage vector," IEEE Trans. Ind. Appl., vol. 38, no. 6, pp. 1572-1580, Nov./Dec. 2002.

[15] M. Botao, L. Congwei, Z. Yang, and L. Fahai, "New SVPWM control scheme for three-phase diode clamping multilevel inverter with balanced dc voltages," in IEEE IECON 2002 Conf., vol. 1, pp. 903-907.

[16] H. L. Liu, N. S. Choi, and G. H. Cho, "DSP based space vector PWM for three level inverter with DC-link voltage balancing," in Proc. IEEE IECON 1991 Conf., vol. 2, pp. 197-203.

[17] S. Busquets-Monge, S. Somavilla, J. Bordonau, and D. Boroyevich, "A novel modulation for the comprehensive neutral-point balancing in the three level NPC inverter with minimum output switching-frequency ripple," in Proc. IEEE-PESC Conf., Jun. 20-25, 2004, vol. 6, pp. 4226-4232.

[18] J. H. Seo and C. H. Choi, "Compensation for the neutral-point potential variation in three level space vector PWM," in Proc. IEEE-APEC Conf., 2001, vol. 2, pp. 1135-1140.
[19] H. Zhang, A. Von Jouanne, S. Dai, A. K. Wallace, and F. Wang, "Multilevel inverter modulation schemes to eliminate common-mode voltages," IEEE Trans. Ind. Appl., vol. 36, no. 6, pp. 1645-1653, Nov.-Dec. 2000.

[20] A. R. Beig, "Application of three level voltage source inverters to voltage fed and current fed high power induction motor drives," Ph.D. dissertation, Indian Inst. Sci., Bangalore, India, 2004.

[21] Reference Guide TMS320F/C240 DSP Controllers: Peripheral Library and Specific Devices Texas Instruments, 1999.

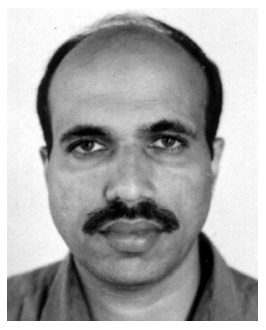

Abdul Rahiman Beig (M'92) received the B.E. degree in electrical and electronics engineering from National Institute of Technology Karnataka, Suratkal, India, in 1989, the M.Tech. and Ph.D. degrees in electrical engineering from Indian Institute of Science, Bangalore, in 1998 and 2004, respectively.

He is currently an Assistant Professor in the Department of Electrical Engineering, National Institute of Technology Karnataka, Suratkal, India. From 1989 to 1992, he was with M/S Kirloskar Electric Company, Ltd., Mysore, India, as a Research and Development Engineer in the drives group. His research interests include ac drives, multilevel inverters.

Dr. Beig received the Innovative Student Project Award for his Ph.D. work from the Indian National Academy of Engineering in 2005 and L\&T- ISTE National Award for the his M.E. thesis from the Indian Society for Technical Education in 1998.

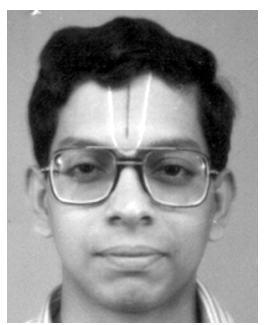

G. Narayanan (S'99-M'01) received the B.E. degree from Anna University, Madras, in 1992, and the M.Tech. degree from the Indian Institute of Technology, Kharagpur, in 1994. He received the $\mathrm{Ph} . \mathrm{D}$. degree from the Indian Institute of Science, Bangalore, in 2000.

$\mathrm{He}$ is currently an Assistant Professor in the Department of Electrical Engineering, Indian Institute of Science, Bangalore. His research interests include ac drives, pulsewidth modulation, multilevel inverters, and protection of power devices.

Dr. Narayanan received the Innovative Student Project Award for his Ph.D. work from the Indian National Academy of Engineering in 2000 and the Young Scientist Award from the Indian National Science Academy in 2003.

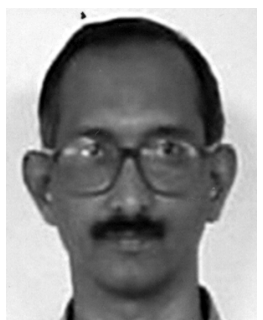

V. T. Ranganathan (SM'84) received the B.E. and M.E. degrees in electrical engineering from the Indian Institute of Science (I.I.Sc.), Bangalore, and the $\mathrm{Ph} . \mathrm{D}$. degree from Concordia University, Montreal, QC, Canada.

He joined the Electrical Engineering Department, I.I.Sc., in 1984, where he is currently a Professor. His research interests are in the area of power electronics and motor drives. He has published several papers in the areas of vector control of ac drives, PWM techniques, split phase induction motor drives, and rotor side control of slip ring induction motors. He is also a consultant to industry in the above areas and has participated in a number of projects.

Dr. Ranganathan received the Prize Paper Award of the IEEE-IAS Static Power Converter Committee and the Tata Rao Prize of the Institution of Engineers, India. He is a Fellow of the Institution of Engineers, India and the Indian National Academy of Engineering. 